\title{
Effect of task-specific training on Eph/ephrin expression after stroke
}

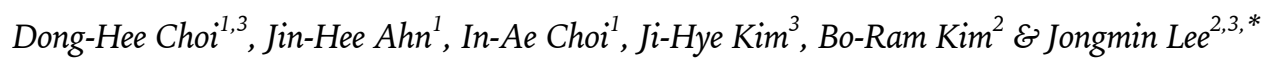 \\ Departments of ${ }^{1}$ Medical Science, ${ }^{2}$ Rehabilitation Medicine, Konkuk University School of Medicine, ${ }^{3}$ Center for Neuroscience Research, \\ Institute of Biomedical Science and Technology, Konkuk University, Seoul 05029, Korea
}

Recent evidence indicates that the ephrin receptors and ephrin ligands (Eph/ephrin) expression modulate axonal reorganization and synaptic plasticity in stroke recovery. To investigate the effect of task-specific training (TST) on Eph/ephrin expression in the corticospinal tract (CST) after stroke, we compared Eph/ephrin expression in the peri-infarct cortex, pyramid, and spinal cord of a photothrombotic stroke model of rat brains treated with or without TST. The TST treatment showed significantly better recovery in the behavioral tests compared with no treatment. The significant upregulation of ephrin-A1 and ephrin-A5 observed in activated astrocytes of the CST at 2 weeks' post-stroke was decreased by TST. At 5 weeks, post-stroke, the elevated ephrin-A5 levels were decreased in the ipsilateral pyramid and spinal cord by TST. Glial fibrillary acidic protein was upregulated concomitantly with the altered ephrin expression after stroke, and the expression of these proteins was attenuated by TST. These data suggest that TST alters the expression of ephrin ligands in the CST after stroke. [BMB Reports 2016; 49(11): 635-640]

\section{INTRODUCTION}

The brain shows a limited capacity for spontaneous repair of motor paralysis after stroke (1). Rehabilitative training has been shown to be a promising intervention to enhance long-term outcomes in patients with stroke, whereas electrical stimulation and pharmacological treatments have shown promise in animal models (1). Although rehabilitation is well known to improve functional outcomes, the mechanism underlying motor recovery after stroke remains unclear. Various mechanisms, including functional reorganization of perilesional

*Corresponding author. Tel: +82-2-2030-5345; Fax: +82-2-20307899; E-mail: leej@kuh.ac.kr

https://doi.org/10.5483/BMBRep.2016.49.11.172

Received 9 October 2016, Revised 14 October 2016, Accepted 17 October 2016

Keywords: Eph/ephrin expression, Reactive astrocytes, Stroke, Stroke recovery, Task-specific training regions (2), restoration of the damaged lateral corticospinal tract (CST) (3), rewiring of the contralateral CST $(4,5)$, and contributions by other motor areas (2-4). have been proposed. Axonal sprouting after stroke arises in peri-infarct areas, a region of cortical remapping associated with restoration after stroke in humans $(6,7)$. In the axons, restorative regeneration and outgrowth are inhibited by an axonal growth inhibitor, myelin-associated proteins, formation of a perineuronal net, the glial scar formed at the injury site, and the expression of repulsive cues for growth cones (e.g., ephrins and semaphorins) $(8,9)$.

The Eph/ephrin family represents the largest family of tyrosine kinases (10). Within the central nervous system, the role of Eph/ephrin signaling has mainly been identified as providing axon guidance during development (11) and neural plasticity in adults (12). Several studies have identified the Eph/ephrin family as growth inhibitory proteins expressed after neuronal injury. A recent study demonstrated that ephrin-A5 is upregulated in reactive astrocytes after stroke (13). Astrocytic ephrin-A5 blocks axonal regeneration in the peri-infarct cortex and limits motor recovery after stroke (13). In addition, the EphA2 receptor and its high-affinity ligands, ephrin-A1, -A2, and $-A 3$, directly contribute to the disruption of the blood-brain barrier and neuronal loss after ischemic injury (10). By contrast, activation of ephrin-A3 reverse signaling regulates astrocytic glutamate uptake under ischemic conditions (14). The reduction of EphA4 enhances motor function after stroke in EphA4 $4^{-1-}$ mice (9). Moreover, neuronal EphA4 receptor expression in axons of the CST of the spinal cord preserves midline crossing of lateral CSTs by ephrin-B3, a repulsive signal in axon guidance, expressed in myelinating oligodendrocytes of the mouse spinal cord $(8,15,16)$.

Recently, Wahl and colleagues demonstrated that Nogo-A antibody therapy enhances motor recovery via CST plasticity after stroke $(1,17)$. We previously reported that the functional recovery afforded by task-specific training (TST) after stroke might vary depending on lesion size and the timing of training. The mechanism underlying this functional recovery may involve contralesional CST plasticity and transcallosal axonal sprouting (4). Several studies have focused on the role of the ephrin receptors and ligands in the peri-infarct area after stroke, whereas the expression of Eph/ephrin has not been 
widely studied in the CST after stroke. Therefore, the aims of this study were to identify possible therapeutic targets of the Eph/ephrin family and to modulate CST plasticity and enhance stroke recovery. We demonstrated that TST altered the expression of Eph/ephrin in the CST after stroke. Controlling the expression of Eph/ephrin and TST appears to be a promising therapeutic strategy to improve recovery from stroke.

\section{RESULTS}

TST improved recovery of motor function after stroke To determine the effects of TST on the expression of Eph/ephrin, we first examined the effects of TST on functional recovery after stroke using the single pellet reaching task (SPRT). A detailed timeline for the experiment is provided (Fig. 1A). Fig. $1 \mathrm{~B}$ and $1 \mathrm{C}$ show the successful reaches throughout

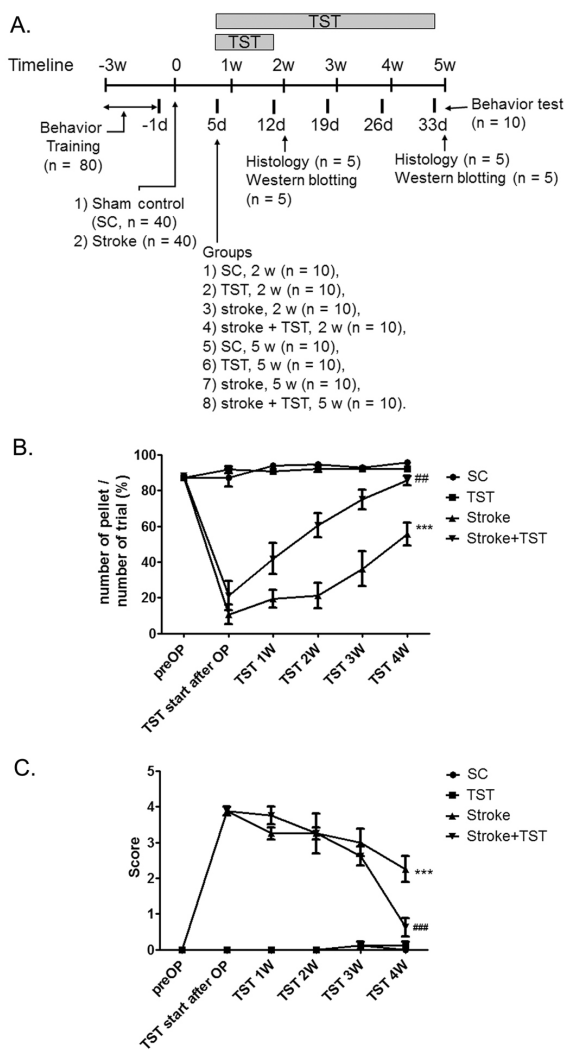

Fig. 1. The timeline of the experiment and recovery of motor function by TST after photothrombotic stroke. (A) In a study of the effect of TST on the expression of Eph/ephrin, SC and stroke rats with or without TST were used for behavior test, protein expression studies, and immunohistochemical analysis. (B, C) TST improved recovery of motor function after stroke using by SPRT and parallel-bar walking test. $* * * \mathrm{P}<0.001$ vs SC (sham control), ${ }^{\# \#} \mathrm{P}<0.05,{ }^{\# \# \# P} \mathrm{P} 0.001$ vs Stroke. Results are presented as the mean \pm SEM, $n=10$ group. the course of the experiment. The reaching ability of each rats was assessed at 1, 2, 3, and $4 \mathrm{w}$ after stroke with or without TST initiated at 5 days after stroke. Stroke injury produced significant impairment in all groups compared with the controls $(P<0.001)$. The group that received TST after stroke exhibited an improved reaching ability from 5 days after TST compared with stroke control group (Fig. 1B, different from the stroke control; $\mathrm{P}<0.001)$. The repeated measures ANOVA detected significant effects of group $(\mathrm{F} 3,30=30.38$; $\mathrm{P}<$ 0.001 ) and time $(F 5,150=46.71 ; \mathrm{P}<0.001)$, and $\mathrm{a}$ significant interaction of group $\mathrm{x}$ time $(\mathrm{F} 15,150=17.68$; $\mathrm{P}<$ 0.001 ). At $1,2,3$, and 4 weeks, there was a pronounced improvement in the groups that underwent TST after stroke ( $P$ $<0.001$ ) (Fig. 1B). Fig. 1C shows the mean number of foot faults when traversing the elevated narrow beam. The nonparametric Friedman analysis detected significant main effects of group (chi-square $=13.77 ; \mathrm{P}<0.001$ ). The TST after stroke group made significantly fewer foot faults per step than did the stroke control group (Fig. 1C; $\mathrm{P}<0.001$ ).

\section{Effect of TST on the expression of Eph/ephrin in protein extracts of the CST after stroke}

To determine the effects of TST on the expression of Eph/ephrin, we immunoblotted protein extracts and performed immunostaining in peri-infarct cortical tissues and ipsilateral pyramid and spinal cord tissues. Ephrin-A1, ephrin-A5, and Glial fibrillary acidic protein (GFAP) levels in the ipsilateral pyramid were significantly increased after stroke compared with the control pyramid. These increased levels of expression were then decreased by TST for $1 \mathrm{w}$ at 5 days after stroke (Fig. 2A). In the spinal cord, EphA2 and EphA4 expression levels were increased after stroke; EphA2 levels were then decreased after TST for $1 \mathrm{w}$, and the elevated levels of EphA4 remained unaltered after TST. Ephrin-A1, ephrin-A5, and GFAP expression levels were increased in the ipsilateral spinal cord and were then attenuated by TST (Fig. 2B). We also confirmed the effect of TST for $4 \mathrm{w}$ on the expression of ephrin-A1, ephrin-A2, ephrin-A5, and GFAP in the ipsilateral CST after stroke. Ephrin-A2 expression was increased at $5 \mathrm{w}$ after stroke and remained unaltered subsequently by TST for $4 \mathrm{w}$ in the ipsilateral pyramid (Fig. 2C). The levels of ephrin-A5 expression were increased at $5 \mathrm{w}$ in the ipsilateral pyramid and spinal cord after stroke (Fig. 2D). These increased ephrin-A5 levels in the ipsilateral pyramid and spinal cord were attenuated by TST for $4 \mathrm{w}$ from 5 days after stroke (Fig. 2C and 2D). GFAP expression was dramatically increased in the CST after stroke and was then significantly decreased by TST for 4 $w$ in the CST (Fig. 2C and 2D).

\section{Effect of TST on the expressions of ephrin-A1 and ephrin-A5 in the CST after stroke}

To determine the cell-type specificity of the expression of ephrin-A1 and ephrin-A5 in the CST after stroke, we performed immunohistochemistry using brain tissues from the cortex, 

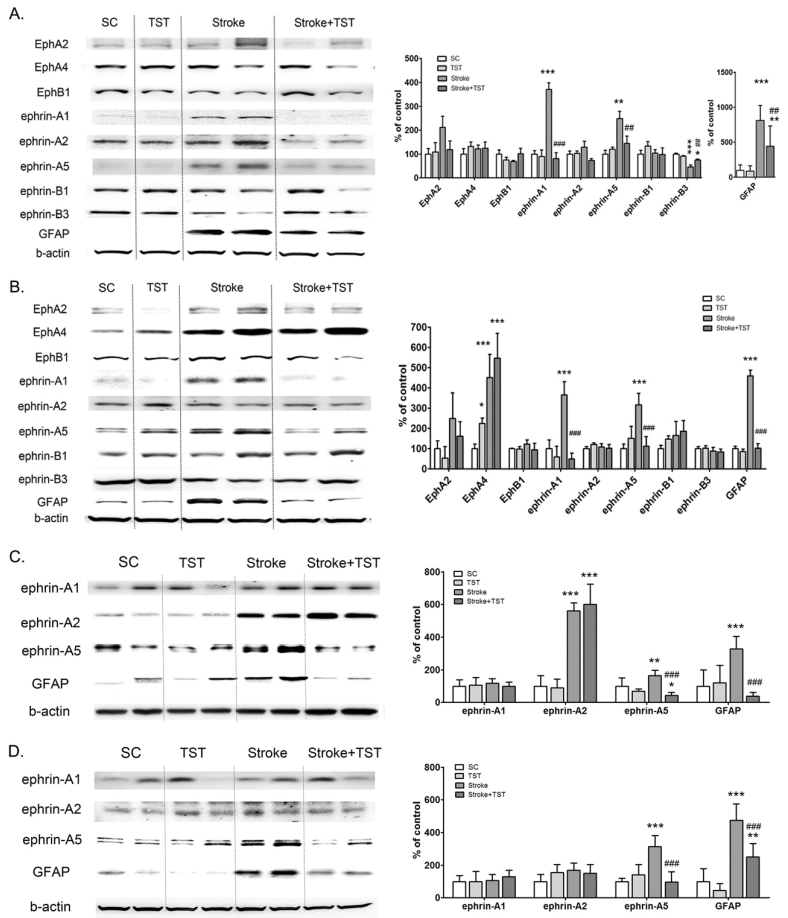

Fig. 2. Effect of TST for 1 week and 4 weeks on the expression of Eph/ephrin in protein extracts of CST after stroke. Representative photomicrographs of western blots for Eph/ephrin, GFAP, and $\beta$-actin in total lysates of the ipsilateral pyramid and spinal cord at $1 \mathrm{w}(\mathrm{A}, \mathrm{B})$ and $4 \mathrm{w}(\mathrm{C}, \mathrm{D})$ after stroke with or without TST initiated at 5 days after stroke. Signal intensities were measured using Quantity One software and are shown as a percentage of control. $\beta$-Actin, internal control. Results are presented as the mean $\pm \mathrm{SD}, \mathrm{n}=5$. ${ }^{* * \mathrm{P}}<0.01, * * * \mathrm{P}<0.001$ vs SC; ${ }^{\# \#} \mathrm{P}<$ 0.01, \#\# $\mathrm{P}<0.001$ vs Stroke.

pyramid, and spinal cord. Ephrin-A1 and ephrin-A5 expression levels in the ipsilateral cortex of the stroke group were significantly increased at $2 \mathrm{w}$ after stroke compared with that observed in the sham-treated control group (Supplementary Fig. $1 \mathrm{~A}$ and $1 \mathrm{~B}$ ). Ephrin-A1 immunostaining (green) was mostly colocalized with neurons (NeuN, red) stained with Torpo-3 (nuclei, blue) (as identified by the white staining observed after merging the three images). However, astrocytes did not express ephrin-A1, which was demonstrated by coimmunostaining using anti-GFAP (astrocytes) and anti-ephrin-A1 antibodies. Ephrin-A5 immunostaining (green) was mostly colocalized with astrocytes (GFAP), which were immunostained red in the cortex (as identified by the yellow staining observed after merging the two images). However, neurons did not express ephrin-A5, which was demonstrated by coimmunostaining using anti-NeuN (neurons) and antiephrin-A5 antibodies. These results suggest the neuron-specific expression of ephrin-A1 and the astrocyte-specific expression of ephrin-A5 in the ipsilateral cortex. Although the increased

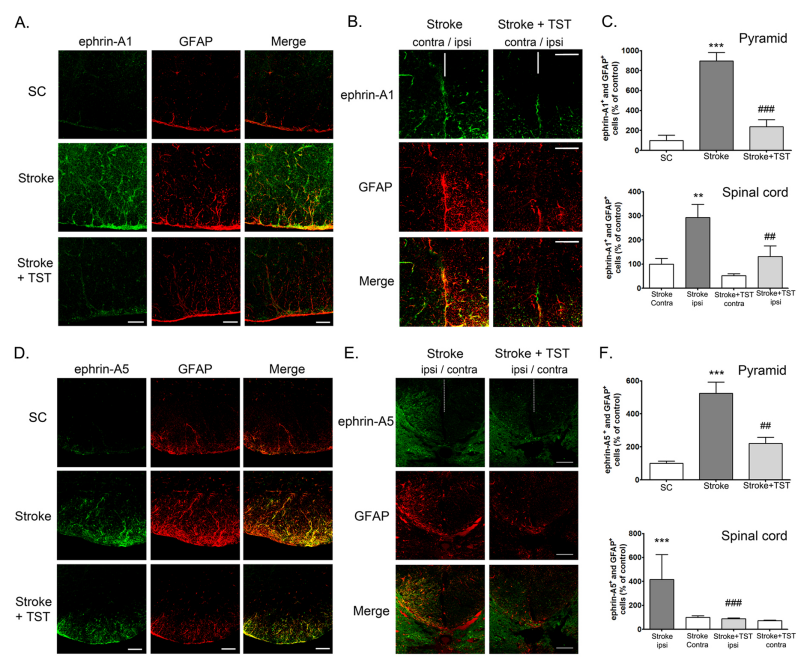

Fig. 3. Effect of TST on the expressions of ephrin-A1 and ephrin-A5 in the pyramid and spinal cord after stroke. Representative photomicrograph of ephrin-A1 (A, B) or -A5 (D, E) and GFAP (red) immunostaining in the pyramid (A, D) and spinal cord $(B, E)$ regions at 2 weeks after stroke. Immunostaining of ephrin-A1 or -A5 (green) and GFAP (red) in astrocyte cells in the ipsilateral pyramid and spinal cord. (C, F) Counts of ephrin-A1- or ephrinA5- positive astrocytes of total cells in the ipsilateral pyramid and spinal cord. Results are presented as the mean \pm SEM, $\mathrm{n}=5$ /group. ${ }^{* * \mathrm{P}}<0.05,{ }^{* *} \mathrm{P}<0.001$ vs SC or Stroke contra; ${ }^{\#} \mathrm{P}<0.01,{ }^{\# \#} \mathrm{P}<0.001$ vs Stroke or Stroke ipsi. Scale bars $=50 \mu \mathrm{m}$. contra: contralateral region; ipsi: ipsilateral region; white dotted line: spinal cord midline.

levels of neuronal ephrin-A1 observed in the ipsilateral cortex of the stroke group were decreased by TST for 1 week after the stroke, ephrin-A5 expression remained unaltered by TST. Astrocyte activation was attenuated by TST (Supplementary Fig. 1B). We also found that ephrin-A1 and ephrin-A5 expression in the ipsilateral pyramid and spinal cord of the stroke group was profoundly increased at 2 and 5 weeks after stroke operation compared with that observed in CST of the sham control group, respectively (Fig. 3 and 4). Ephrin-A1 and ephrin-A5 immunostaining (green) was mostly colocalized with activated astrocytes (GFAP), which were immunostained red in the CST (as identified by the yellow staining observed after merging the two images). Coimmunostaining which was demonstrated by coimmunostaining using anti-Tuj-1 (neurons) and anti-ephrin-A1 or -ephrin-A5 antibodies showed that neurons did not express ephrin-A1 and ephrin-A5, (data not shown). These results suggest the activated-astrocyte-specific expression of ephrin-A1 and ephrin-A5 in the SCT. The increased ephrin-A1 and ephrin-A5 expression observed in the activated astrocytes of the ipsilateral CST was decreased by TST for 1 week (Fig. 3) and 4 weeks (Fig. 4) after stroke, respectively. GFAP-positive astrocyte activation was significantly decreased in TST-treated animals (Fig. 3 and 4). 


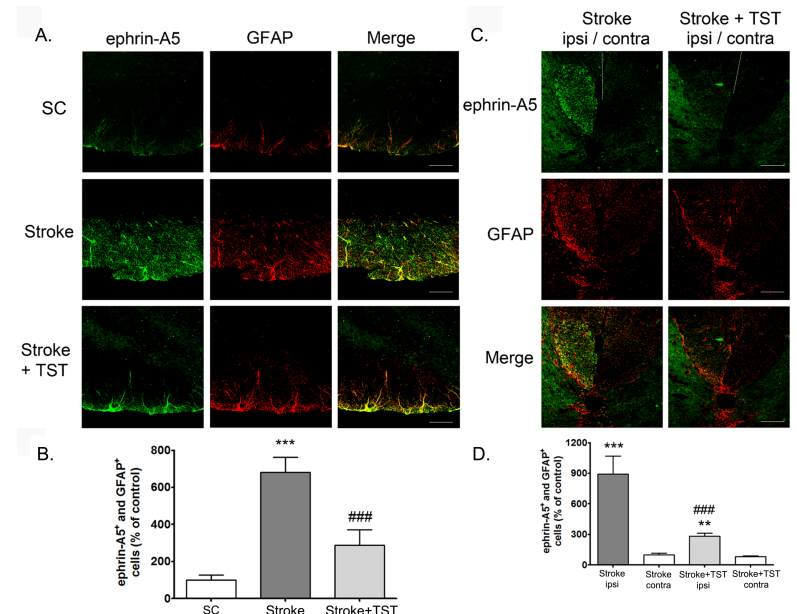

Fig. 4. Effect of TST for 4 weeks on the expressions of ephrin-A5 in the pyramid and spinal cord after stroke. Representative photomicrograph of ephrin-A5 immunostaining in the pyramid (A) or spinal cord (C) regions of CST at 5 weeks after stroke. Scale bars $=50 \mu \mathrm{m}$. Counts of ephrin-A5-positive astrocytes of total cells in the ipsilateral pyramid (B) and spinal cord (D). Results are presented as the mean \pm SEM, $\mathrm{n}=$ 5/group. $* * * \mathrm{P}<0.001$ vs. SC or Stroke.

\section{DISCUSSION}

In this study, we showed the presence of spatiotemporal changes in the expression of Eph/ephrin in the peri-infarct cortex and CST after cerebral cortical photothrombotic stroke. To elucidate whether the TST of the damaged forelimb contributes to the changes in Eph/ephrin expression observed in the ipsilateral CST after stroke, we compared spatiotemporal changes in the expression of Eph/ephrin between stroke and stroke + TST rats. Axonal sprouting occurs in peri-infarct regions after stroke (18). Neuronal injury induces expression of the growth-regulatory genes, including growth-promoting and growth-inhibiting genes (18). The location of and balance between the expression of these genes have decisive effects on the degree of axonal sprouting (18).

The Eph/ephrin family includes growth-associated inhibiting factors (10). Elevated ephrin-A5 in activated astrocytes mediates inhibition of axonal sprouting in peri-infarct areas and motor recovery after stroke (13). These results are in close agreement with our findings of an increase in ephrin-A5 expression in reactive astrocytes in the peri-infarct cortex after stroke. Additional findings showed that ephrin-A5 expression is also elevated in reactive astrocytes of the pyramid and spinal cord after stroke. The EphA2 receptor and its ephrin-A1, -A2, and -A3 ligands induced dysfunction of the blood-brain barrier and apoptotic cell death in an experimental model of stroke (10). Our findings demonstrate that the expression of ephrin-A1 was increased in neurons of the peri-infarct cortex and in activated astrocytes of the CST after stroke. Up- regulation of Eph/ephrin on astrocytes potentially mediates astrocytic gliosis around the lesion site in the brain injury, which secrete extracellular matrix molecules such as chondroitin sulfate proteoglycans, which are known for their inhibitory effect on axonal growth and regeneration (19). This astrocyte reactivity was also associated with neuronal death in the area adjacent to the lesion site $(10,19)$.

Task-oriented therapy is important in the functional recovery after stroke. The best way to relearn a given task is to train specifically for that task (20). TST can produce cortical reorganization and associated, meaningful functional improvements (20). We reported previously that functional recovery after stroke was enhanced by TST, the underlying mechanism of which may involve contralesional CST plasticity and transcallosal axonal sprouting (4). A recent report demonstrated that TST increases the number of cortical neurons which projecting to the cervical cord and CST fibers from the cortex in both the gray matter and dorsal column of the spinal cord (21).

Several studies have focused on the role of the Eph/ephrin in the peri-infarct cortical region after stroke, whereas its role in the CST, one of the largest fiber pathways associated with forelimb motor function, has not been widely studied. To our knowledge, for the first time, we demonstrated spatiotemporal changes in the expression of Eph/ephrin in the CST after stroke with or without TST treatment. Our data suggest that the spatiotemporal changes in ephrin-A1 and -A5 expression observed in the peri-infarct cortex and CST after stroke are variable, and that these changes may be reversed by rehabilitation therapy such as TST. Reduced astrocyte activation by TST may involve in downregulation of ephrin expression in astrocytes after stroke. One possible mechanism has been reported that upregulated expression of ephrin-A4 in the astrocytes via $\mathrm{Ca}^{2+} /$ calmodulin-dependent protein kinase II/CAMP responsive element binding Protein (CaMKII/CREB) pathway $(22,23)$. Further studies are needed to elucidate the signaling mechanisms of TST effect on the regulation of Eph/ephrin expression after stroke. Moreover, inhibition of ephrin-A1 and -A5 expression by TST may represent a mechanism that improves neurological function. Therefore, regulation of ephrin-A1 and -A5 expression by TST may be a potential therapeutic target for brain repair after stroke.

\section{MATERIALS AND METHODS}

\section{Animals}

A total of 80 male Wistar rats ( 8 weeks of age, weighing 288 \pm 2.75 g; Orent Bio Inc., Seongnam, Korea; 40 sham control rats (SC) and 40 photothrombotic-ischemia-operated rats, Stroke) were used in this study. The animals were raised in a temperature-controlled room $\left(23 \pm 0.5^{\circ} \mathrm{C}\right)$ with $12 \mathrm{~h}: 12 \mathrm{~h}$ light/dark cycle. All experimental procedures were approved by the Animal Experiment Review Board of Institutional Animal Care and Use Committee (IACUC) of Konkuk University 
(Permit Number: KU13123). Animal treatments, including anesthesia and euthanasia, were carried out in accordance with the Principle of Laboratory Animal Care (NIH publication No. 85-23, revised 1985). They were also in accordance with the Stroke Therapy Academic Industry Roundtable (STAIR) criteria for preclinical stroke investigations (24).

\section{Photothrombotic ischemia surgery}

Focal cortical infarcts were produced by focusing light on the sensorimotor and motor cortices in Rose Bengal-treated rats (4, $25)$. Briefly, the rats were anesthetized with ketamine (50 $\mathrm{mg} / \mathrm{kg}$ ) and xylazine $(5 \mathrm{mg} / \mathrm{kg}$ ) mixture through intraperitoneal (i.p.) injection and were then placed in a stereotaxic frame (Stoelting Co., Wood Dale, IL, USA). The skull was exposed, and the fiber optic bundle of a KL1500 LCD cold light source (Carl Zeiss, Jena, Germany) with a 4-mm aperture was positioned on the skull at the bregma and $4.0 \mathrm{~mm}$ lateral to the midline over the right sensorimotor and motor cortices. The photochemical dye Rose Bengal (Sigma-Aldrich, St. Louis, $\mathrm{MO}, \mathrm{USA})$ was infused via i.p. injection for $5 \mathrm{~min}(20 \mathrm{mg} / \mathrm{kg})$, after which the light was switched on for $20 \mathrm{~min}$. Sham control animals were subjected to exposure to light without Rose Bengal i.p. injection for 20 min.

\section{Single-pellet reaching test}

The single-pellet reaching test was used as described previously (4). A successful reach occurred when an animal grasped a food pellet (Bioserve Inc., Frenchtown, NJ, USA), transported it using its paw to the cage, and placed it in its mouth. Success (\%) was defined as the number of pellets obtained/20 × 100 .

\section{Parallel-bar walking test}

Parallel bar testing ( $n=10$ /group) is particularly sensitive to the hind limb coordination impairment and was performed as described previously (4). The number of errors made per meter in $1 \mathrm{~min}$ were calculated and scored.

\section{Task-specific training}

Five days after the photothrombotic surgery, TST groups were exposed to daily training (5 days a week for 1 week or 4 weeks) as described previously (4). Animals reached their impaired forepaw through the slot and ate pellets (Bioserve Inc, Beltsville, MD, USA). Three hundred pellets were placed on the right side of the reclined plate, and animals used their impaired forepaw to eat the pellets. Animals were in the training box for $20 \mathrm{~min}$ or until they ate all of the pellets.

\section{Western blot analysis}

The peri-infarct cortices, pyramid, and spinal cord tissues $(\mathrm{n}=$ 5/group) were washed with ice-cold PBS and lysed on ice in RIPA buffer $(50 \mathrm{mM}$ Tris- $\mathrm{HCl} \mathrm{pH} 7.4,150 \mathrm{mM} \mathrm{NaCl}, 1 \%$ NP40, $0.25 \%$ Na-deoxycholate, and $0.1 \%$ SDS) containing a protease inhibitor mixture and phosphatase inhibitors (Sigma-
Aldrich, St. Louis, MO, USA). Thirty micrograms of soluble protein were subjected to SDS-PAGE and electrotransferred onto a PVDF membrane (26). Specific protein bands were detected using specific Eph/ephrin antibodies (Supplementary Table 1) and enhanced chemiluminescence (Pierce, Rockford, IL, USA).

\section{Double-fluorescence immunostaining of tissues}

Double-fluorescence staining was performed as described previously (4). Free-floating sections $(40 \mu \mathrm{m})$ were incubated in $0.1 \mathrm{M}$ PBS containing 5\% normal donkey serum and $0.3 \%$ Triton $\mathrm{X}-100$ for $1 \mathrm{~h}$, and subsequently incubated overnight with the specific primary antibodies (Supplementary Table 1) in 2\% normal donkey serum (Vector Laboratories, Burlingame, CA, USA) in PBS at $4^{\circ} \mathrm{C}$ and incubated with a 1:200 dilution of Alexa Fluor-conjugated donkey anti-rabbit (488) or donkey anti-mouse (546) antibodies (Invitrogen, Grand Island, NY, USA) for $1 \mathrm{~h}$ at room temperature and mounted on glass slides using Vectashield (Vector Laboratories, Burlingame, CA, USA). Fluorescent signals were evaluated on a confocal microscope (LSM 710, Carl Zeiss, Oberkochen, Germany).

\section{Quantitative analysis}

Sections including the cortex, pyramid, and spinal cord from 5 rats per group were subjected to analysis. Five regions of interest (ROIs) of $0.1 \mathrm{~mm}^{2}$ per one section from the cortex, pyramid, and spinal cord were selected. The number of ephrin-A1- and GFAP-; or NeuN, ephrin-A5-, and GFAP-; or NeuN-positive cells was counted in each ROI and averaged. Data are represented as the percentage of total cells. All quantitative analyses were carried out in a blind manner.

\section{Data analysis and statistics}

The SPRT was analyzed using a two-way repeated-measures analysis of variance (ANOVA), followed by a post hoc least significant differences multiple comparisons test. The parallelbar walking test was analyzed using a nonparametric Friedman analysis of variance, followed by a post hoc Dunnett's T3 test. A one-way ANOVA was used to compare the intensity of Western blot results and cell counts after immunostaining, between groups. This statistical analysis comprised a one-way ANOVA followed by a Newman-Keuls multiple comparisons test. All data were expressed as the mean \pm standard deviation. Null hypotheses of no differences were rejected if $\mathrm{P}<0.05$. All data analyses were performed using the SPSS version 20.0 software (IBM Corporation, New York, USA).

\section{ACKNOWLEDGEMENTS}

This research was supported by Basic Science Research Program through the National Research Foundation of Korea(NRF) funded by the Ministry of Science, ICT and future Planning (NRF-2014R1A2A1A11050236 grant to DHC, NRF2014R1A2A1A11050248, and NRF-2012R1A1A4A01013749 
grant to JL), a grant from the Korea Health Technology R\&D Project through the Korea Health Industry Development Institute (KHIDI), funded by the Ministry of Health \& Welfare, Republic of Korea (grant number: HI14C2339), and the National Research Foundation of Korea (NRF) grant funded by the Korea government (NRF-2016R1A5A2012284). The funders had no role in study design, data collection, and analysis, decision to publish, or preparation of the manuscript.

\section{CONFLICTS OF INTEREST}

The authors have no conflicts of interest to declare.

\section{REFERENCES}

1. Wahl AS, Omlor W, Rubio JC et al (2014) Neuronal repair. Asynchronous therapy restores motor control by rewiring of the rat corticospinal tract after stroke. Science $344,1250-1255$

2. Murphy TH and Corbett D (2009) Plasticity during stroke recovery: from synapse to behaviour. Nat Rev Neurosci $10,861-872$

3. Jang SH (2009) A review of the ipsilateral motor pathway as a recovery mechanism in patients with stroke. NeuroRehabilitation 24, 315-320

4. Lee KH, Kim JH, Choi DH and Lee J (2013) Effect of task-specific training on functional recovery and corticospinal tract plasticity after stroke. Restor Neurol Neurosci 31, 773-785

5. Liu Z, Li Y, Zhang X, Savant-Bhonsale S and Chopp M (2008) Contralesional axonal remodeling of the corticospinal system in adult rats after stroke and bone marrow stromal cell treatment. Stroke 39, 2571-2577

6. Carmichael ST (2006) Cellular and molecular mechanisms of neural repair after stroke: making waves. Ann Neurol $59,735-742$

7. Lo EH (2008) A new penumbra: transitioning from injury into repair after stroke. Nat Med 14, 497-500

8. Benson MD, Romero MI, Lush ME, Lu QR, Henkemeyer $M$ and Parada LF (2005) Ephrin-B3 is a myelin-based inhibitor of neurite outgrowth. Proc Natl Acad Sci U S A 102, 10694-10699

9. Lemmens $R$, Jaspers $T$, Robberecht $W$ and Thijs $V N$ (2013) Modifying expression of EphA4 and its downstream targets improves functional recovery after stroke. Hum Mol Genet 22, 2214-2220

10. Thundyil J, Manzanero S, Pavlovski D et al (2013) Evidence that the EphA2 receptor exacerbates ischemic brain injury. PLoS One 8, e53528

11. Flanagan JG (2006) Neural map specification by gradients. Curr Opin Neurobiol 16, 59-66

12. Yamaguchi $Y$ and Pasquale EB (2004) Eph receptors in the adult brain. Curr Opin Neurobiol 14, 288-296
13. Overman JJ, Clarkson AN, Wanner IB et al (2012) A role for ephrin-A5 in axonal sprouting, recovery, and activitydependent plasticity after stroke. Proc Natl Acad Sci U S A 109, E2230-2239

14. Yang J, Luo X, Huang X, Ning Q, Xie M and Wang W (2014) Ephrin-A3 reverse signaling regulates hippocampal neuronal damage and astrocytic glutamate transport after transient global ischemia. J Neurochem 131, 383-394

15. Yokoyama N, Romero MI, Cowan CA et al (2001) Forward signaling mediated by ephrin-B3 prevents contralateral corticospinal axons from recrossing the spinal cord midline. Neuron 29, 85-97

16. Kullander K, Croll SD, Zimmer M et al (2001) Ephrin-B3 is the midline barrier that prevents corticospinal tract axons from recrossing, allowing for unilateral motor control. Genes Dev 15, 877-888

17. Moses ZB, Abd-El-Barr MM and Chi JH (2015) Timing is everything in corticospinal tract recovery after stroke. Neurosurgery 76, N18-19

18. Carmichael ST, Archibeque I, Luke L, Nolan T, Momiy J and Li S (2005) Growth-associated gene expression after stroke: evidence for a growth-promoting region in periinfarct cortex. Exp Neurol 193, 291-311

19. Goldshmit Y and Bourne J (2010) Upregulation of EphA4 on astrocytes potentially mediates astrocytic gliosis after a cortical lesion in the marmoset monkey. J Neurotrauma 27, 1321-1332

20. Bayona NA, Bitensky J, Salter K and Teasell R (2005) The role of task-specific training in rehabilitation therapies. Top Stroke Rehabil 12, 58-65

21. Okabe N, Shiromoto T, Himi N et al (2016) Neural network remodeling underlying motor map reorganization induced by rehabilitative training after ischemic stroke. Neuroscience [Epub ahead of print]

22. Wu LY, Yu XL and Feng LY (2015) Connexin 43 stabilizes astrocytes in a stroke-like milieu to facilitate neuronal recovery. Acta Pharmacol Sin 36, 928-938

23. Choi JK, Park SY, Kim KH, Park SR, Lee SG and Choi BH (2014) GM-CSF reduces expression of chondroitin sulfate proteoglycan (CSPG) core proteins in TGF-beta-treated primary astrocytes. BMB Rep 47, 679-684

24. Feinklestein SP, Fisher M, Furland AJ et al (1999) Recommendations for standards regarding preclinical neuroprotective and restorative drug development. Stroke 30, 2752-2758

25. Watson BD, Dietrich WD, Busto R, Wachtel MS and Ginsberg MD (1985) Induction of reproducible brain infarction by photochemically initiated thrombosis. Ann Neurol 17, 497-504

26. Kim YJ, Kim JE, Choi HC, Song HK and Kang TC (2015) Cellular and regional specific changes in multidrug efflux transporter expression during recovery of vasogenic edema in the rat hippocampus and piriform cortex. BMB Rep 48, 348-353 